\title{
Methicillin-resistant Staphylococcus aureus metrics for patients in Saudi Arabia
}

\author{
Asghar Nazeer ${ }^{1}$ and Jaffar A. Al-Tawfiq ${ }^{2}$ \\ Epidemiology Services Unit ${ }^{1}$ and Specialty Internal Medicine ${ }^{2}$, Dhahran Health Center, Saudi Aramco Medical \\ Services Organization, Saudi Aramco, Dhahran, Saudi Arabia
}

\begin{abstract}
Introduction: The World Health Organization recommends essential measures to "combat drug resistance", including instituting surveillance "everywhere". Standardized metrics are crucial for reliable surveillance. Studies publish metrics with varying definitions for multi-drug resistant organisms (MDRO). The Society for Healthcare Epidemiology of America (SHEA) and the Healthcare Infection Control Practices Advisory Committee (HICPAC) proposed standardized metrics for MDRO for consistent reporting, identifying high-risk groups, and evaluating interventions.

Methodology: We retrieved 73 studies through PubMed using the search terms "methicillin-resistant Staphylococcus aureus", "MRSA", and "Saudi Arabia". We selected 20 studies that reported MRSA incidence or prevalence in patients and/or percentage among Staphylococcus aureus isolates and evaluated these metrics against the closest matching SHEA/HICPAC metrics.

Results: We outlined issues applicable to MRSA metrics such as comparison of risk-unadjusted metrics; their pooling for different hospitals; not accounting for post-discharge infections; non-specification of AST-based, and healthcare and community associated infections' related, standardized metrics by SHEA/HICPAC; and appropriate temporal criteria for nosocomial infections. We elaborated salient features of reviewed metrics versus their SHEA/HICPAC complements. Terminology and definitions of reviewed metrics differed from SHEA/HICPAC counterparts. Some did not satisfy the epidemiological or statistical criteria for their reported category; e.g. prevalence indicators were classified as incidence and vice versa.

Conclusions: SHEA/HICPAC metrics would be useful for future studies. Our results show an imminent need for an international consensus on fundamental MDRO surveillance metrics; illustrate surveillance scenarios requiring standardized metrics; identify some indicators from Saudi studies supplementing SHEA/HICPAC metrics; and underscore SHEA/HICPAC's advice for avoiding comparison of risk-unadjusted metrics between hospitals.
\end{abstract}

Keywords: hospitals; incidence; prevalence; methicillin-resistant Staphylococcus aureus; review; Saudi Arabia.

J Infect Dev Ctries 2012; 6(3):223-233.

(Received 13 August 2011 - Accepted 05 October 2011)

Copyright () 2012 Nazeer and Al-Tawfiq. This is an open-access article distributed under the Creative Commons Attribution License, which permits unrestricted use, distribution, and reproduction in any medium, provided the original work is properly cited

\section{Introduction}

The World Health Organization (WHO) recommended six essential measures to "combat drug resistance" on World Health Day 2011 [1], emphasizing that "surveillance is crucial and should be rapidly put in place everywhere" [1]. Standardized metrics are essential for reliable surveillance and evaluating effectiveness of preventive interventions [2]. Published studies employ varying terms, definitions, and metrics for infections detected in healthcare facilities, such as hospital-acquired [3], healthcare-acquired [4], community-acquired [5], and nosocomial [2] infections, etc. with the suffix "associated" [6] or "onset" [7] used instead of "acquired" in some contexts. The Society for Healthcare Epidemiology of America (SHEA) and Healthcare Infection Control Practices Advisory
Committee (HICPAC) recommended standardized metrics for multi-drug resistant organisms (MDRO) in healthcare settings for consistent usage [2].

SHEA/HICPAC proposed two epidemiological classifications of MDRO infections by their likely transmission setting [2]. The first relies on time since admission [2]. MDRO infection is termed "community-onset" if detected within three calendar days since admission and "hospital-onset" afterward. The second classification employs temporal and clinical criteria [2]. MDRO infection is considered "nosocomial" if there is no evidence that infection was incubating or present on admission and it is detected after a specified time cutoff [2], healthcareassociated if attributable to current or recent healthcare delivery, medical devices, or procedures [2], or community-associated without known 
healthcare-associated risk factors or link to recent healthcare delivery [2]. SHEA/HICPAC posited metrics related to each of these classifications [2].

Several studies reported methicillin-resistant Staphylococcus aureus (MRSA) metrics, e.g., percentage of MRSA among all Staphylococcus aureus (S. aureus) isolates, MRSA incidence, and/or prevalence for patients attending Saudi hospitals. These studies were done prior to the publication of SHEA/HICPAC metrics [2]. Guidance for standardization of MDRO metrics was scant until then [8]. The definitions of infections and colonizations by transmission setting and MRSA metrics consequently varied among those studies. This variance is avoidable in the future. SHEA/HICPAC MDRO metrics include indices for antibiotic susceptibility, incidence, and prevalence. We summarize the related MRSA metrics extracted from the published papers [9-27] and a letter [28] from Saudi Arabia (all referred to as studies hereafter), compare them with the relevant SHEA/HICPAC indicators [2], discuss their pros and cons, and offer our conclusions.

The aim of this review is to heighten awareness about the SHEA/HICPAC standardized metrics for MDRO in developing countries by employing them as benchmarks for evaluating the MRSA metrics reported by studies of patients in Saudi Arabia. We anticipate that by doing so, future MRSA and MDRO studies in developing countries will be more likely to avoid the current variability in terminology, definition, and calculation of such indicators by employing the applicable SHEA/HICPAC metrics for facilitating uniform comprehension of their reported metrics by readers. A secondary aim is to identify some valid and useful metrics for quantifying healthcare-associated or community-associated MRSA incidence or prevalence reported by the reviewed studies, which may complement the set of SHEA/HICPAC metrics. SHEA/HICPAC did not specify any corresponding standardized metrics, even though healthcare-associated and communityassociated infections are discrete categories in their epidemiological classification of MDRO. This is an opportune juncture for such a review given that the WHO dedicated World Health Day 2011 to "combat drug resistance", and adoption of standardized metrics for surveillance of MDRO is an integral part of the strategy for reporting and fighting them.

\section{Methodology}

Seventy-three studies were identified through a PubMed search as of April, 2011, using the keywords "MRSA", "methicillin-resistant Staphylococcus aureus", and "Saudi Arabia". Studies that reported percentage of MRSA among S. aureus isolates or incidence and/or prevalence of MRSA that matched with SHEA/HICPAC metrics exactly or with certain relaxations, and those providing data for calculating such metrics, were selected. Twenty studies [9-28] reported the sought indicators or relevant data. For multi-year studies, metrics for the latest year were chosen $[11,15,18,19,20]$ except when the published paper provided only an average or range for the study period [14,16,17,26,27]. SHEA/HICPAC metrics [2] were used as benchmarks for appraising reviewed MRSA indicators. The pros and cons of the extracted MRSA metrics were identified in comparison with SHEA/HICPAC metrics and useful reported metrics complementing them were highlighted.

\section{Results}

Table 1 has four sections based on the clinical presentations of MRSA relevant to reported metrics, and columns for SHEA/HICPAC metrics [2] reported by reviewed studies or estimated from their data. Incidence and prevalence rates are per 100 admissions and densities per 1,000 patient-days.

Table 1 lists metrics reported by reviewed studies in the regular font, and those calculated from their data in italics. Reported metrics not meeting SHEA/HICPAC's specifications [2] or epidemiological or statistical criteria for their reported classification were placed in the most closely related SHEA/HICPAC category [2] and underlined. The justification is given in the discussion. Pooled values or range of values for multi-hospital studies are listed in the bold font.

\section{Discussion}

We employed SHEA/HICPAC metrics as a framework for highlighting the pros and cons of the published MRSA metrics to inform authors doing similar studies. However, we appreciate the usefulness of MRSA metrics proposed by other organizations as well $[29,30]$. SHEA/HICPAC concedes that many issues are unsettled in defining and validating the most practical and useful MDRO metrics [2]. We aim to motivate further debates on MRSA metrics for accelerating an international consensus [31]. 


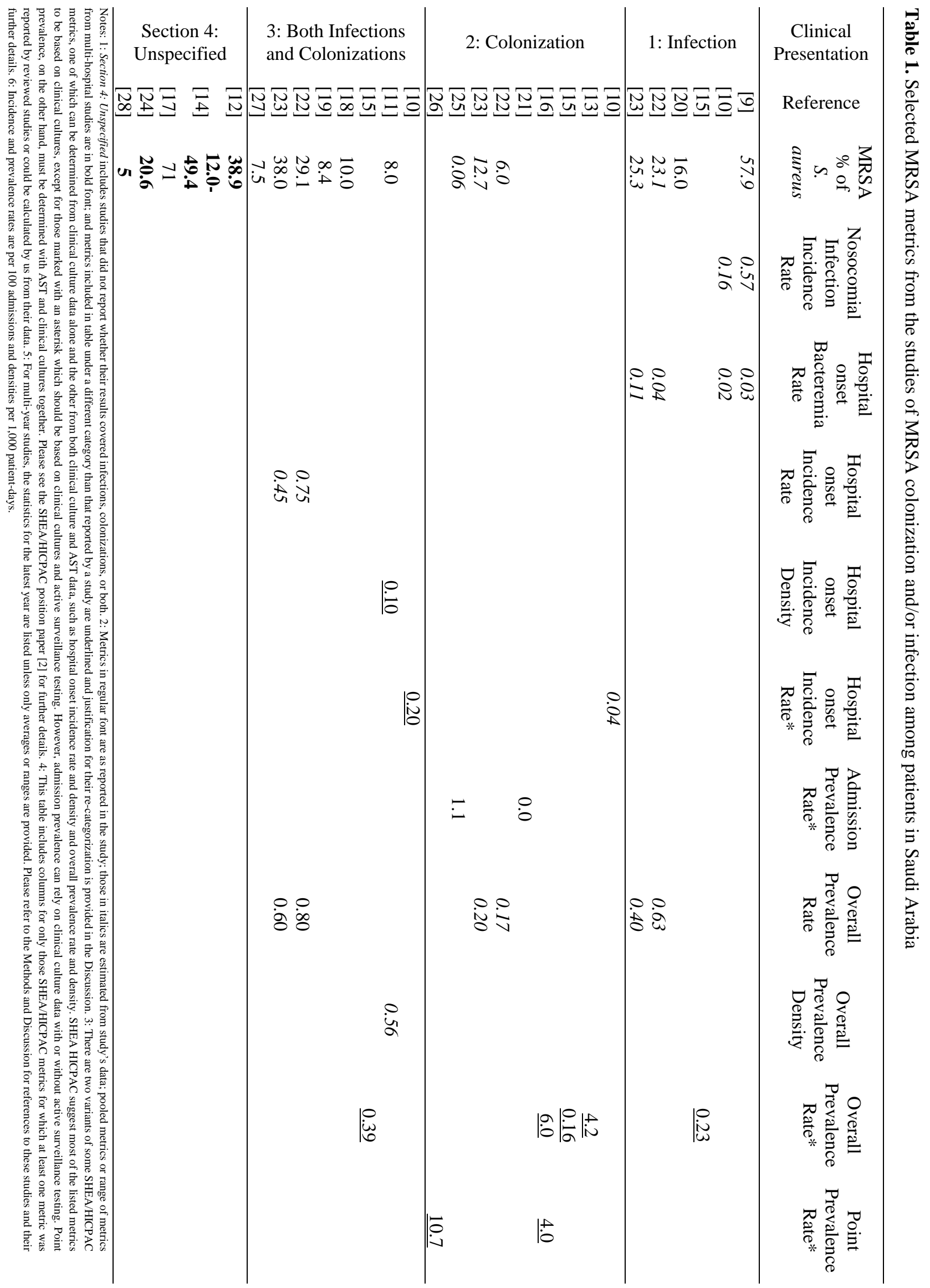


The discussion hereafter is organized in two subsections. The first focuses on overarching issues and the second on comparing the reviewed indicators with closest matching SHEA/HICPAC metrics. Finally, we give concluding remarks.

\section{Overarching issues}

We discuss here the aspects of the reviewed studies, the SHEA/HICPAC position paper [2], and contextual references broadly affecting the interpretation of the reviewed metrics. SHEA/HICPAC selected metrics based on their utility and simplicity for gauging MDRO occurrence in hospitals and evaluating intervention's effectiveness [2]. They provided a set of fundamental metrics and not a compendium. We highlight some other metrics reported by the published studies but do not imply they are indispensable for SHEA/HICPAC to standardize and include in their repertoire.

\section{Significance of clinical presentation of MRSA}

MRSA metrics are grouped in Table 1 by the related clinical presentations such as colonizations, infections, both, or otherwise unspecified.

Studies capturing both infections and colonizations [10,11,15,18,19,22,23,27] assess the "exposure burden" and the "colonization pressure" for healthcare acquisition of MRSA [2,32]. Both infection and colonization predispose to future MRSA infections. MRSA infections were detected in $29 \%$ of patients within 18 months of previous colonization or infection [2,33]. Studies for separately assessing infections $[9,10,15,20,22,23]$ and colonizations $[10,13,16,21-23,25,26]$ are also informative, as each has distinct implications. MRSA infections cause high morbidity, mortality, disability, and healthcare costs [34,35]. Antibiotic use in infected patients leads to selective pressure [36] facilitating emergence of more widely resistant strains, e.g., to vancomycin [37]. Colonization facilitates MRSA spread [32,38]. Decolonization may reduce transmission [39-41], but the effectiveness of decolonization therapy is uncertain $[42,43]$. Four studies reported metrics separately for infections and colonizations [10,15,22,23]. Three of these studies reported high "infection-to-colonization ratio" $[10,22,23]$ and two of them stated that AST is essential for accurate estimation of that ratio [22,23].

\section{Comparisons of risk unadjusted metrics}

SHEA/HICPAC advised against inter-facility comparisons without valid risk adjustment, even for their standardized MRSA metrics [2]. Many reviewed studies compared their MRSA indicators with others in their discussions without considering the disparity in the patient, study, and hospital characteristics. For instance, case-mix differed among the studies. Among the twenty studies reviewed, thirteen dealt with inpatients [9-13,15,17-19,21-23,25], two with inpatients and outpatients, [14,20] and two with special patient groups such as cystic fibrosis [16] or renal failure patients [26]. Three studies did not specify whether they obtained isolates from inpatients, outpatients, or both [24,27,28]. Not all studies stated employing Centers for Disease Control and Prevention's (CDC) National Healthcare Safety Network's (NHSN) [47] infection definitions. The definitions of MRSA infections by transmission settings differed. These variables confound comparisons of MRSA metrics. Nevertheless, three studies directly compared metrics among hospitals $[12,14,22]$. One study compared percentages of MRSA among S. aureus isolates for four [12] and the other for six hospitals [14]. The third [22] compared MRSA metrics for two hospitals. The metrics for one hospital [22] are listed in one row, and corresponding metrics for the other hospital reported in this study [22] as well as another study by the same first author [23] in the next row in Table 1.

\section{Pooled MRSA metrics}

One study [24] gave a collective estimate of the MRSA percentage among $S$. aureus isolates for four hospitals in Makkah and another [28] for five hospitals in Riyadh. Two multi-hospital studies provided MRSA percentages among $S$. aureus isolates for each hospital [12,22]. One of these [12] reported combined MRSA percentage among S. aureus isolates for four participating hospitals, which was $38.9 \%$ as listed in Table 1. It also published the values for every participating hospital that were 41.5, $37.5,40.3$, and $36.3 \%$ [12]. The other study [22] reported separate metrics for each hospital. Since the metrics for one of the hospitals in this study [22] were also reported in another study by the same authors, they are listed in Table 1 with reference to that study [23]. Separately reported metrics for each facility in a multi-hospital study are more informative than pooled statistics. SHEA/HICPAC advised against comparing MRSA metrics for different facilities without risk-adjustment [2]. We similarly caution that pooling risk-unadjusted metrics from hospitals with disparate characteristics may be 
problematic without a methodically sound metaanalysis. On the other hand, pooling data over time for the same facility, in which marked changes in hospital or patient characteristics have not occurred during the study period, to determine averages or ranges may be useful. However, time trends are obscured in such summary measures.

\section{Post-discharge infections}

Excluding post-discharge infections underestimates nosocomial infections ascribed to an admission episode. Jain et al. attributed MRSA infections detected in readmitted patients by AST within 48 hours of discharge to discharging unit [44]. One reviewed study [9] included post-discharge surgical site infections (SSI) reported by surgeons. Most reviewed studies did not report whether postdischarge infections were taken into account.

\section{Incidence and prevalence metrics}

The distinction between incidence and prevalence metrics is subtle in some studies, but it is important. Healthcare-associated incidence rates, reported by a study of infections or colonizations attributable to current and recent hospitalizations and prior medical or surgical interventions [15], are more aptly classifiable as healthcare-associated prevalence rates. MRSA prevalence reported by a study for new colonizations and nosocomial infections detected after 72 hours of admission, or related to an intervention during hospital stay, [10] is theoretically an incidence rate. Some studies used the terms "incidence" [11] or "prevalence" [14,22,23] for the percentage of MRSA among $S$. aureus isolates. These terms are best reserved for metrics pertaining to patient populations.

\section{Healthcare-associated MRSA metrics}

A study reported healthcare-associated incidence rates for colonizations and infections. [15] It employed temporal and clinical criteria similar to the SHEA/HICPAC healthcare-associated infection classification [2], for which they did not propose standardized metrics [2]. The study's healthcareassociated infections incidence rate [15], more appropriately interpreted as healthcare-associated prevalence rate (see above), is tentatively placed under overall prevalence rate as are its other metrics due to unavailability of an appropriate placement in Table 1. However, overall prevalence encompasses both healthcare-associated and community-associated infections. Another study reported healthcare- associated isolates (non-duplicate) per 100,000 patient-days for infections and colonizations associated with prior healthcare delivery or certain medical devices and detected within 48 hours of admission [11]. This indicator can be designated as community-onset healthcare-associated MRSA prevalence density [2,45]. We did not list this indicator [11] in Table 1, as SHEA/HICPAC did not specify any comparable metric. However, they stated that a subset of community-onset infections could be categorized as healthcare-associated if data were available to decipher the role played by healthcare facilities in the potential transmission of MDRO [2]. These two studies, and the ones related to community-associated infections noted below, underscore the need for relevant SHEA/HICPAC standardized metrics.

\section{Community-associated infections}

These are a public health issue $[11,19,20,45,46]$ and contribute to admission prevalence and "exposure burden" [2] in hospitals. SHEA/HICPAC did not define standardized metrics for communityonset and community-associated infections. Therefore, the relevant metrics from the reviewed studies $[9,11,18-20,22,23]$ cannot be compared with any SHEA/HICPAC counterparts. However, the percentages of MRSA among $S$. aureus isolates from some studies of MRSA as a community pathogen $[18,19,20]$ are listed in Table 1 as they have a comparable SHEA/HICPAC metric of antibiogram.

\section{Appropriate time cutoff}

SHEA/HICPAC exhorted researchers to assess the effects of different definitions of epidemiological classification of infections on the associated metrics and MDRO surveillance results [2]. They acknowledged that the best specification of the time cutoff for discriminating between community-onset versus hospital-onset and community-associated from nosocomial infections was still an open research question [2]. Furuya et al. noted that two versus three calendar days and 48- versus 72-hour cutoffs did not affect SHEA/HICPAC defined MRSA incidence rates in two smaller hospitals, but a cutoff of two versus three days made significant difference for the largest among three participating hospitals [8]. Five reviewed studies used 48 hours $[9,11,15,18,20]$ and four 72 hours as cutoff points $[10,19,22,23]$. Two of them are of interest. A 30-month study considered MRSA as community-acquired if the isolate was obtained within 72 hours of admission and was 
unrelated to a hospital intervention [19]. A concurrent 36-month study for that hospital defined MRSA as community-acquired if culture isolates were positive within 48 hours of admission [18]. The number of community-acquired isolates remained 20 , despite the studies' different time cutoffs and durations $[18,19]$, though the percentages of community-acquired among MRSA isolates in these studies were $22 \%$ [18] and $18.2 \%$ due to their unequal denominators [19].

\section{Standardized metrics based on AST}

The Clinical Laboratory Standards Institute (CLSI) recommends antibiograms based on clinical cultures [48]. SHEA/HICPAC endorsed this advice for antibiogram proposed as their MDRO susceptibility monitoring metric [2]. They recommended clinical cultures, with or without AST, for their other metrics [2]. However, they posited no metric based on AST alone. AST-based metrics extracted from several reviewed studies, e.g., percentage of MRSA among S. aureus isolates [25], point prevalence, [26] admission prevalence, [21] colonization incidence, [10] and period prevalence [13], are useful for some surveillance scenarios and worthy of consideration by SHEA/HICPAC for standardization and adoption if deemed appropriate.

\section{AST's utility in detecting MRSA}

AST at admission helps confirm MRSA acquisitions during current admission [2]. Incidence rates combining information from clinical cultures and AST better quantify new MRSA acquisitions [2,49]. Jain et al. employed universal AST at admission, history of MRSA infection or colonization in the past 12 months, and clinical cultures to detect new MRSA transmission [44]. A reviewed study included AST on admission for high-risk patients besides routine clinical cultures [15], but it did not report how many patients had AST-based evidence for MRSA acquisition. Another study employed AST (timing unspecified) and clinical cultures 72 hours or more after admission [10]. Both studies did not assess additional MRSA colonizations or infections identified by AST that routine clinical cultures missed [10,15]. AST identified about 90\% and clinical cultures $10 \%$ of MRSA carriers in the study by Jain et al. [44], which testifies to the significant importance of AST in detecting MRSA carriers for prevention of transmission.

\section{AST's impact on metrics}

AST is useful for detecting additional colonizations [2,50] and infections [8]. Identifying more MRSA colonizations and infections by AST may affect MRSA metrics differentially. Furuya et al. reported that AST significantly affected prevalence but not incidence [8], leading to a $14 \%$ increase in patients identified with hospital-onset MRSA infection or colonization and $78 \%$ increase in prevalence of MRSA colonization or infection [8].

These observations are supported by the findings that AST increased detection and reduced misclassification of prevalent as incident cases by more than 17\% [51-52]. Two reviewed studies used AST and clinical cultures but did not quantity AST's impact on reported metrics $[10,15]$.

\section{Following CLSI guidelines}

CLSI and SHEA/HICPAC stipulate that the resistance rate should be calculated as the number of intermediate and resistant strains divided by the total number of isolates [2,48]. Adhering to the CLSI guidelines is important, as variations in classifying resistant isolates affect the accuracy of MRSA metrics. Most of the reviewed studies followed their guidance. One study included the few strains with intermediate susceptibility among the susceptible strains [17]. In that study, the average percentage of MRSA among $S$. aureus isolates from clinical cultures of obstetric specimens for a three-year period was $71 \%$. This high value is striking, even though the study included intermediate resistant isolates among susceptible. Four of the reviewed studies did not explicitly report following CLSI guidelines $[9,16,17,27]$.

\section{Challenges of applying SHEA/HICPAC metrics}

Furuya et al. [8] provided valuable insights regarding challenges of applying the SHEA/HICPAC metrics to a real-world setting and the efforts required for manual and electronic data collection and calculating selected metrics. They also tested some propositions for further research by SHEA/HICPAC [2] regarding evaluating different definitions of nosocomial incidence, the effect of excluding patient-days not-at-risk from denominators, and whether to look at one, two, or three years' worth of prior data when including or excluding patients with history of MRSA infection or colonization for calculating some metrics such as nosocomial incidence rates [8]. 


\section{Proxy versus exact metrics}

Most of the SHEA/HICPAC metrics are "proxy" metrics [2] and not "exact" measures. Huang [51] examined the benefits and limitations of proxy metrics versus exact measures, especially in relation to the national recommendations for healthcareassociated infections and related metrics including those proposed by SHEA/HICPAC. The paper is useful in understanding the pros and cons of the proxy measures for employing them judiciously [51].

Comparing the reviewed metrics with SHEA/HICPAC standardized metrics

The discussion henceforth is organized according to SHEA/HICPAC categories for standardized metrics [2] we used as benchmarks for evaluating the reviewed metrics. We provide explanations for our arbitrary reclassification and placement of underlined metrics in Table 1 wherever relevant.

\section{Monitoring susceptibility patterns}

Antibiogram: SHEA/HICPAC specified antibiogram as a metric for monitoring MDRO susceptibility patterns [2]. Antibiograms give the percentage of susceptible isolates among those tested [48]. We listed in Table 1 the percentages of MRSA among $S$. aureus isolates, as reported by the reviewed studies $[9,11,12,14,17-19,20,22-24,27,28]$ to focus on the occurrence of resistant strains. Notably, the listed percentages of MRSA among S. aureus isolates are not a homogenous group. Some were pooled estimates from multi-hospital studies [24,28]; others were for a single hospital $[9,11,12,17,18,20,22,23,27]$; and one study gave a range for six hospitals [14]. The study durations, inclusion of infections and/or colonizations, and transmission settings differed. Some studies reported percentages of MRSA among $S$. aureus isolates by transmission setting $[11,18,19]$. SHEA/HICPAC did not define distinct metrics for monitoring susceptibility patterns for infections, colonizations, or both based on AST and/or clinical cultures by transmission setting. An AST-based study assessed the percentage of MRSA colonizations among patients within eight hours of admission [25]. We derived the percentage of MRSA among S. aureus isolates obtained from patients. This metric was tentatively placed under antibiograms in Table 1, as it assesses MRSA susceptibility patterns as do antibiograms. However, SHEA/HICPAC has defined no comparable AST-based metrics for quantifying MDRO susceptibility in consistency with CLSI's recommendation for basing antibiograms only on clinical cultures $[2,48]$. .

\section{Estimating infection burden}

Hospital-onset MRSA bacteremia rate and density: The reviewed studies did not report any of these metrics, recommended as "basic" by SHEA/HICPAC [2]. These are well-validated metrics robust to variations in clinical practices [2,51], and are reliable measures for evaluating the impact of interventions for reducing healthcare-associated infections [53,54]. We estimated hospital-onset bacteremia rates from the data provided in four reviewed studies $[9,10,22,23]$ and listed them in Table 1.

Nosocomial MRSA incidence or incidence density rate: No study reported these rates. We estimated nosocomial MRSA incidence rate from two studies [9,10]. SHEA/HICPAC recommended clinical cultures for obtaining microbiological data for calculating these metrics, as was done by one study [10]; the other study employed both AST and clinical culture [9]. As discussed above, AST improves detection and correct classification of new MRSA infections attributable to the current admission [2,8,44,49,51-52], which is important for more accurate estimation of nosocomial MRSA incidence rate and density.

Device-associated MRSA infections incidence density rate and SSI rate

No study reported device-associated MRSA infections incidence density or SSI rate. MRSA is a common cause of ventilator-associated pneumonia, central venous catheter associated bloodstream infections, and SSI $[44,55,56]$. Four studies reported the numbers of nosocomial SSI caused by MRSA $[9,10,22,23]$ but not the number of surgical procedures for calculating SSI rates.

\section{Estimating exposure burden}

Overall prevalence or prevalence density rate based on clinical culture data: Two studies reported overall prevalence based on clinical cultures for infections and colonizations combined [22,23]. We calculated the clinical culture based prevalence separately for infections and colonizations from their data [22,23]. These studies were overlapping, as one comparing two hospitals [22] included the hospital covered by the other study [23] for the same time period. From another study, we calculated the overall prevalence density of colonizations and infections 
based on clinical cultures [11]. This five-year study mentioned that MRSA screening of admitted patients with AST was discontinued since 2002 and admissions with previous history of MRSA colonization or infection were always considered MRSA cases [11]. SHEA/HICPAC recommended including patients with previous history of colonizations and infections for calculating overall prevalence and prevalence density rates [2]. This study had no data about such patients [11], so we could not take it into account for calculating the overall prevalence density statistic for the last year of this five-year study (Table 1). Two other studies also did not mention if such patients were accounted for while calculating overall prevalence [22,23].

Overall prevalence or prevalence density rate based on AST and clinical culture data: No study reported this metric. However, AST-based MRSA prevalence was reported for a five-day study of adult and pediatric patients [13]. This indicator does not match SHEA/HICPAC defined metrics for overall prevalence, as the study was AST-based, while SHEA/HICPAC defined those metrics based on clinical cultures alone or clinical cultures plus AST. Moreover, the study population comprised both adults and children, while SHEA/HICPAC metrics for adult acute-care hospitals have not been validated for pediatric patients [2]. MRSA metrics of period prevalence for hospitals serving adult and pediatric patients need to be evaluated and standardized as such hospitals are more common than those catering solely to adult populations.

The prevalence of colonization within one to six months of follow-up was reported in one study [16]. This metric is tentatively listed under the overall prevalence rate in Table 1 to include this metric for information, though it does not encompass both infections and colonizations as required by SHEA/HICPAC for overall prevalence metrics. This study [16] furthermore did not examine prevalence for a defined calendar time but retrospectively for a variable follow-up period since diagnosis or enrollment. Prevalence density would have been a preferable metric for this study, as it adjusts for variable follow-up period using person-time of observation as the denominator.

Admission prevalence rate based on clinical culture data with or without AST: SHEA/HICPAC specified clinical cultures with or without AST for determining admission prevalence. No study met these criteria for data sources. An AST-based study reported $0 \%$ admission prevalence of MRSA colonization in ophthalmic care patients within 48 hours of admission [21]. A more favorable case-mix among ophthalmic care patients compared with those encountered in an acute-care hospital for this threeweek study, and excluding patients with short stays, those who refused, and for whom nasal cultures were not obtained within 48 hours, are factors that might have led to an absence of colonization among subjects selected for this study [21].

Point prevalence rate based on point prevalence surveys: No study reported this SHEA/HICPAC metric for prevalence [2] measured at a given point in calendar time based on AST and clinical culture. The metrics for colonization prevalence among patients at the time of an ongoing enrollment for two studies $[16,26]$ cannot be regarded as point prevalence, though we listed them in Table 1 as such, as no metric was specified by SHEA/HICPAC for a point in care from a cohort perspective. Nasopharyngeal aspirates and sputum samples were obtained from cystic fibrosis patients at the time of diagnosis in one study [16]. In the other study, five nasal swabs were taken from end-stage renal disease patients on enrollment into outpatient hemodialysis [26].

\section{Quantifying healthcare acquisition}

Incidence or incidence density rate of hospitalonset MDRO based on clinical culture data: One study reported "nosocomial isolates per 100,000 patient-days" [11]. This metric had non-duplicate MRSA isolates obtained more than 48 hours after admission as numerator and patient-days as denominator. SHEA/HICPAC requires applying CDC/NHSN infection definitions as clinical criteria for estimating the organism-specific nosocomial infection incidence rate and density [2]. The metric relied only on temporal criteria and did not employ above-referred clinical criteria for nosocomial infections. It also encompassed isolates related to both infections and colonizations [11]. It is thus more akin to hospital-onset MRSA incidence density [2], as that SHEA/HICPAC metric is only temporal criteria based and may include both newly acquired colonizations and infections during current admission. However, this study [11] did not exclude patients with history of MRSA infection or colonization which is a SHEA/HICPAC requirement for accurate characterization of this metric for quantifying healthcare acquisition of MDRO.

One study reported prevalence of "nosocomial" or "hospital-acquired" infections and colonizations [10]. We considered it as hospital-onset incidence 
rate, as this SHEA/HICPAC metric includes infections and colonizations acquired during current admission. SHEA/HICPAC requires applying CDC/NHSN infection definitions for its MDRO nosocomial infection rate [2], which this study did. From the study data [10], we estimated this MRSA metric and listed it in Table 1. We also calculated the hospital-onset incidence rate for colonizations and listed it [10]. SHEA/HICPAC only requires clinical cultures for estimating MDRO nosocomial infection rate, but this study also employed AST. AST enhances detection of infections and especially asymptomatic colonizations, [2,8,50] and together with clinical cultures improves the accuracy of MDRO metrics by proper classification of infections by transmission setting [51-52]. Hence AST usage in this study is advantageous.

\section{Concluding remarks}

We affirm that this review is not intended to be definitive or exhaustive. We could only express our views about a few aspects of the metrics reported by each reviewed study and admit that an in-depth discourse on this perplexing subject is beyond our purview. We just endeavored to stimulate discussions to examine MRSA metrics more thoroughly for informing future studies by underscoring the perplexity created by varying definitions and calculation methods of reported MRSA metrics from studies done in Saudi Arabia and highlighting their pros and cons by employing SHEA/HICPAC standardized MDRO metrics as benchmarks.

In essence, we undertook this review to achieve the following objectives:

1. Compare the MRSA metrics reported in the published studies of patients attending Saudi hospitals with SHEA/HICPAC metrics and evaluate their pros and cons.

2. Underscore the significance of SHEA/HICPAC recommendations for standardized MDRO metrics and the conceptual framework they provide for future studies in Saudi Arabia.

3. Highlight the SHEA/HICPAC's advice to avoid comparing MDRO metrics from different hospitals without valid risk adjustment, which some of the reviewed studies did.

4. Exemplify some reviewed study designs, i.e., the one assessing period prevalence for adult and pediatric patients [13], for which SHEA/HICPAC's metrics need to be validated.
5. Point out useful measures from Saudi studies, e.g., the ones related to healthcare-associated $[11,15]$ and community-associated [18,19,20[ infections, for which SHEA/HICPAC did not suggest any counterparts.

6. Reiterate the need for an international consensus - best achievable under the auspices of the WHO - on standardized metrics for credible surveillance of MDRO.

The main message of this review is to emphasize reporting standardized metrics for MDRO in published studies, such as those proposed by SHEA/HICPAC, to facilitate their comprehension by readers. The definitions of MDRO metrics are continually evolving across the world with different national agencies proposing their own versions $[29,30]$. An international consensus on a comprehensive set of standardized metrics for MDRO reporting worldwide would enable establishment of MDRO surveillance systems employing similar indicators for assessment of the incidence and prevalence of antibiotic resistant organisms across regions and countries. These standardized indicators can be employed for monitoring MDRO as well as evaluating interventions against them. This would be a conducive step in enabling the collaboration of various healthcare systems to "combat antibiotic resistance", an apt theme for the WHO's World Health Day 2011, considering the mounting antibiotic resistance worldwide.

\section{References}

1. World Health Organization: Podcast: World Health Day 2011 - combat drug resistance8 April 2011 (Transcript: Dr Raviglione, M) (http://www.who.int/en/). Last accessed 12 June 2011.

2. Cohen AL, Calfee D, Fridkin SK, Huang SS, Jernigan JA, Lautenbach E, Oriola S, Ramsey KM, Salgado CD, Weinstein RA; Society for Healthcare Epidemiology of America and the Healthcare Infection Control Practices Advisory Committee (2008) Recommendations for metrics for multidrug-resistant organisms in healthcare settings: SHEA/HICPAC Position paper. Infect Control Hosp Epidemiol 29: 901-913.

3. Kaiser ML, Thompson DJ, Malinoski D, Richmond A, Warren DK, Dubberke ER (2011) Epidemiology and risk factors for hospital-acquired methicillin-resistant Staphylococcus aureus among burn patients. J Burn Care Res 32: 429-434.

4. Gould IM (2009) Controversies in infection: infection control or antibiotic stewardship to control healthcareacquired infection? J Hosp Infect 73: 386-391.

5. Balma-Mena A, Lara-Corrales I, Zeller J, Richardson S, McGavin MJ, Weinstein M, Weinstein M, Pope E (2011) 
Colonization with community-acquired methicillin-resistant Staphylococcus aureus in children with atopic dermatitis: a cross-sectional study. Int J Dermatol 50: 682-688.

6. Fridkin SK, Hageman JC, Morrison M, Sanza LT, ComoSabetti K, Jernigan JA, Harriman K, Harrison LH, Lynfield R, Farley MM; Active Bacterial Core Surveillance Program of the Emerging Infections Program Network (2005)Methicillin-resistant Staphylococcus aureus disease in three communities. Active Bacterial Core Surveillance Program of the Emerging Infections Program Network. N Engl J Med 352: 1436-1444.

7. Lessa FC, Mu Y, Davies J, Murray M, Lillie M, Pearson A, Fridkin SK, Emerging Infections Program/Active Bacterial Core surveillance MRSA Investigators and the Health Protection Agency Team (2010) Comparison of incidence of bloodstream infection with methicillin-resistant Staphylococcus aureus between England and United States, 2006-2007. Clin Infect Dis 51: 925-928.

8. Furuya EY, Larson E, Landers T, Jia H, Ross B, Behta M (2011) Challenges of applying the SHEA/HICPAC metrics for multidrug-resistant organisms to a real-world setting. Infect Control Hosp Epidemiol 32: 323-332.

9. Abdel-Fattah MM (2005) Surveillance of nosocomial infections at a Saudi Arabian military hospital for a one-year period. Ger Med Sci3: 1-10.

10. Al-Anazi (2009) Prevalence of MRSA in a teaching hospital in Riyadh, Saudi Arabia. Biomedical Research 20: 7-14.

11. Al-Tawfiq JA (2006) Incidence and epidemiology of methicillin-resistant Staphylococcus aureus infection in a Saudi Arabian hospital, 1999-2003. Infect Control Hosp Epidemiol 27: 1137-1139.

12. Asghar AH and Momenah AM (2006) Methicillin resistance among Staphylococcus aureus isolates from Saudi hospitals. Med Princ Pract 15: 52-55.

13. Austin TW, Austin MA, McAlear DE, Coleman BT, Osoba AO, Thaqafi AO, Lamfon MA (2003) MRSA prevalence in a teaching hospital in Western Saudi Arabia. Saudi Med J24: 1313-1316.

14. Baddour MM, Abuelkheir MM, Fatani AJ (2006) Trends in antibiotic susceptibility patterns and epidemiology of MRSA isolates from several hospitals in Riyadh, Saudi Arabia. Ann Clin Microbiol Antimicrob 5: 30.

15. Balkhy HH, Memish ZA, Almuneef MA, Cunningham GC, Francis C, Fong KC, Nazeer ZB, Tannous E (2007) Methicillin-resistant Staphylococcus aureus: a 5-year review of surveillance data in a tertiary care hospital in Saudi Arabia. Infect Control Hosp Epidemiol 28: 976-982.

16. Banjar HH (2004) Microbiological data of cystic fibrosis patients in a tertiary care center in Saudi Arabia. Kuwait Med J36: 177-181.

17. Bilal NE and Gedebou M (2000) Staphylococcus aureus as a paradigm of a persistent problem of bacterial multiple antibiotic resistance in Abha, Saudi Arabia. East Mediterr Health J6: 948-954.

18. Bukharie HA, Abdelhadi MS, Saeed IA, Rubaish AM, Larbi EB (2001) Emergence of methicillin-resistant Staphylococcus aureus as a community pathogen. Diagn Microbiol Infect Dis 40: 1-4.

19. Bukharie HA and Abdelhadi MS (2001) The epidemiology of methicillin-resistant Staphylococcus aureus at a Saudi university hospital. Microb Drug Resist 7: 413-416.

20. Bukharie HA (2010) Increasing threat of communityacquired methicillin-resistant Staphylococcus aureus. Am J Med Sci 340: 378-381.
21. Islam SI and Moore C (2002) Prevalence of methicillinresistant Staphylococcus aureus and associated risk factors on admission to a specialist care eye hospital. Ann Saudi Med 22: 153-157.

22. Madani TA, Al-Abdullah NA, Al-Sanousi AA, Ghabrah TM, Afandi SZ, Bajunid A (2001) Methicillin-resistant Staphylococcus aureus in two tertiary-care centers in Jeddah, Saudi Arabia. Infect Control Hosp Epidemiol 22: 211-216.

23. Madani TA (2002) Epidemiology and clinical features of methicillin-resistant Staphylococcus aureus in the University Hospital, Jeddah, Saudi Arabia. Can J Infect Dis13: 245-250.

24. Momenah AM, Asghar AH, Bukhari SZ, Azhar EI, Ashi AM, Tayeb MT (2005) Accuracy of current oxacillin sensitivity tests routinely used in hospitals in Western Saudi Arabia. Saudi Med J 26: 584-587.

25. Panhotra BR, Saxena AK, Al Mulhim AS (2005) Prevalence of methicillin-resistant and methicillin-sensitive Staphylococcus aureus nasal colonization among patients at the time of admission to the hospital. Ann Saudi Med 25: 304-308.

26. Saxena AK, Panhotra BR, Chopra R (2004) Advancing age and the risk of nasal carriage of Staphylococcus aureus among patients on long-term hospital-based hemodialysis. Ann Saudi Med 24: 337-342.

27. Zaman R and Dibb WL (1994) Methicillin-resistant Staphylococcus aureus (MRSA) isolated in Saudi Arabia: epidemiology and antimicrobial resistance patterns. J Hosp Infect 26: 297-300.

28. Shibl AM, Tawfik AF, Khan AM, Qadri SM, Imambaccus Y, Ramadan, MA (1994) Re: "Prevalence of methicillinresistant staphylococci in Riyadh, Saudi Arabia and their susceptibility to antimicrobial agents." J Antimicrob Chemother 33: 1255-1257.

29. Health Protection Agency, London, UK. Mandatory surveillance reports. (http://www.hpa.org.uk/web/HPAweb\&HPAwebStandard/H PAweb_C/1233906819629) Last accessed 30 April 2011.

30. Canadian Nosocomial Infection Surveillance Program MRSA Surveillance Protocol. Surveillance for methicillinresistant Staphylococcus aureus (MRSA) in CNISP health care facilities. Version December, 2006 (http://www.phacaspc.gc.ca/nois-sinp/projects/pdf/mrsa_protocol_e.pdf). Last accessed 30 April 2011.

31. Struelens MJ (2009) Guidelines and indicators for methicillin-resistant Staphylococcus aureus control in hospitals: toward international agreement? Curr Opin Infect Dis 22: 337-338.

32. Merrer J, Santoli F, Appere de Vecchi C, Tran B, De Jonghe B, Outin H (2000) "Colonization pressure" and risk of acquisition of methicillin-resistant Staphylococcus aureus in a medical intensive care unit. Infect Control Hosp Epidemiol 21: 718-723.

33. Huang SS and Platt R (2003) Risk of methicillin-resistant Staphylococcus aureus after previous infection or colonization. Clin Infec Dis36: 281-285.

34. Shurland S, Zhan M, Bradham DD (2007) Comparison of mortality risk associated with bacteremia due to methicillinresistant and methicillin-susceptible Staphylococcus aureus. Infect Control Hosp Epidemiol 28: 273-279.

35. Engelmann JJ, Carmeli Y, Cosgrove SE, Fowler VG, Bronstein MZ, Trivette SL, Briggs JP, Sexton DJ, Kaye KS (2003) Adverse clinical and economic outcomes attributable 
to methicillin resistance among patients with Staphylococcus aureus surgical site infections. Clin Infect Dis 36: 5 92-598.

36. Tacconelli E, De Angelis G, Cataldo MA,Pozzi, E., Cauda, $R$ (2008)Does antibiotic exposure increase the risk of methicillin-resistant Staphylococcus aureus (MRSA) isolation? A systematic review and meta-analysis. J Antimicrob Chemother 61: 26-38.

37. Centers for Disease Control and Prevention (2002) Staphylococcus aureus resistant to vancomycin-United States, 2002. MMWR Morb Mortal Wkly Rep 51: 565-567.

38. Williams VR, Callery S, Vearncombe M, Simor AE (2009) The role of colonization pressure in nosocomial transmission of methicillin-resistant Staphylococcus aureus. Am J Infect Control 37: 106-110.

39. Robicsek A, Beaumont JL, Suzanne M, Paule, BS, Donna M, Hacek BS, Thomson RB Jr, Kaul KL, King P, Peterson LR (2008) Universal surveillance for methicillin-resistant Staphylococcus aureus in 3 affiliated hospitals. Ann Intern Med 148: 409-418.

40. Davis KA, Stewart JJ, Crouch HK, Florez CE, Hospenthal DR (2004) Methicillin-resistant Staphylococcus aureus (MRSA) nares colonization at hospital admission and its effect on subsequent MRSA infection. Clin Infect Dis 39: 776-782.

41. Robicsek A, Beaumont JL, Thomson RB Jr, Govindarajan G, Peterson LR (2009) Topical therapy for methicillinresistant Staphylococcusaureus colonization: impact on infection risk. Infect Control Hosp Epidemiol 30: 623-632.

42. Simor AE, Loeb M, CIDS/CAMM Guidelines Committee (2004) The management of infection and colonization due to methicillin-resistant Staphylococcus aureus: A CIDS/CAMM position paper. Can J Infect Dis 15: 39-48.

43. Harbarth S, Dharan S, Liassine N, Herrault P, Auckenthaler R, Pittet D (1999) Randomized, placebo-controlled, doubleblind trial to evaluate the efficacy of mupirocin for eradicating carriage of methicillin-resistant Staphylococcus aureus. Antimicrob Agents Chemother 43: 1412-1416.

44. Jain R, Kralovic SM, Evans ME, Evans ME, Ambrose M, Simbartl LA, Obrosky DS, Render ML, Freyberg RW, Jernigan JA, Muder RR, Miller LJ, Roselle GA (2011) Veterans affairs initiative to prevent methicillin-resistant Staphylococcus aureus infections. N Engl J Med 364: 14191430.

45. Klevens RM, Morrison MA, Nadle J, Petit S, Gershman K, Ray S, Harrison LH, Lynfield R, Dumyati G, Townes JM, Craig AS, Zell ER, Fosheim GE, McDougal LK, Carey RB, Fridkin SK; Active Bacterial Core surveillance (ABCs) MRSA Investigators (2007) For the Active Bacterial Core Surveillance (ABCs) MRSA Investigators. Invasive methicillin-resistant Staphylococcus aureus infections in the United States. JAMA 298: 1763-1771.

46. Carleton HA, Diep BA, Charlebois ED, Sensabaugh GF, Perdreau-Remington F (2004) Community-adapted methicillin-resistant Staphylococcus aureus (MRSA): Population dynamics of an expanding community reservoir of MRSA. J Infect Dis 190: 1730-1738.

47. Horan TC, Andrus M, Dudeck MA (2008) CDC/NHSN surveillance definition of health care-associated infection and criteria for specific types of infections in the acute-care setting. Am J Infect Control 36: 309-332.

48. Clinical and Laboratory Standards Institute (CLSI) (2005) Analysis and presentation of cumulative antimicrobial susceptibility test data: approved guideline. 2nd ed. Vol. 25. No. 28. Wayne, PA: CLSI, M39-A2.

49. Weinstein RA and Kabins SA (1981) Strategies for prevention and control of multiple drug-resistant nosocomial infection. Am J Med 70: 449-454.

50. Salgado CD, Farr BM (2006) What proportion of hospital patients colonized with methicillin-resistant Staphylococcus aureus are identified by clinical microbiological cultures? Infect Control Hosp Epidemiol 27: 116-121.

51. Huang SS (2009) Healthcare-associated infection: Assessing the validity of our measures. Clin Infect Dis 48: 1116-1122.

52. Huang SS, Rifas-Sirman SL, Warren DK, Diekema DJ, Rego VR, Yokoe DS, Platt R; Centers for Disease Control and Prevention Epicenters Program (2007) Improving methicillin resistance Staphylococcus aureus surveillance and reporting in intensive care units. J Infect Dis 195: 330338.

53. Huang SS, Yokoe DS, Hinrichsen VL, Spurchise LS, Datta R, Miroshnik I, Platt R (2006) Impact of routine intensive care unit surveillance cultures and resultant barrier precautions on hospital-wide methicillin-resistant Staphylococcus aureus bacteremia. Clin Infect Dis 43: 971978.

54. Shitrit P, Gottesman BS, Katzir M, Kilman A, Ben-Nissan Y, Chowers M (2006) Active surveillance for methicillinresistant Staphylococcus aureus (MRSA) decreases the incidence of MRSA bacteremia. Infect Control Hosp Epidemiol 27: 1004-1008.

55. Klein E, Smith DL, Laxminarayan R (2007) Hospitalizations and deaths caused by methicillin-resistant Staphylococcus aureus, United States, 1999-2005. Emerg Infect Dis 13: 1840-1846.

56. Hidron AI, Edwards JR, Patel J, Horan TC, Sievert DM, Pollock DA, Fridkin SK; National Healthcare Safety Network Team; Participating National Healthcare Safety Network Facilities (2008) NHSN annual update: antimicrobial-resistant pathogens associated with healthcareassociated infections: annual summary of data reported to the National Healthcare Safety Network at the Centers for Disease Control and Prevention, 2006-2007. Infect Control Hosp Epidemiol 29: 996-101[Erratum, Infect Control Hosp Epidemiol 2009; 30: 107.]

\author{
Corresponding author \\ Dr Jaffar A. Al-Tawfiq \\ PO Box 76 \\ Room A-428-2, Building 61, Dhahran Health Center \\ Saudi Aramco, Dhahran 31311, Saudi Arabia \\ Telephone: +966-3-877-3524 \\ Fax: +966-3-877-3790 \\ Email: jaffar.tawfiq@aramco.com;jaltawfi@yahoo.com
}

Conflict of interests: No conflict of interests is declared. 